\title{
KANTOROVICH-RUBINSTEIN NORM AND ITS APPLICATION IN THE THEORY OF LIPSCHITZ SPACES
}

\author{
LEONID G. HANIN
}

(Communicated by Palle E. T. Jorgensen)

\begin{abstract}
We obtain necessary and sufficient conditions on a compact metric space $(K, \rho)$ that provide a natural isometric isomorphism between completion of the space of Borel measures on $K$ with the Kantorovich-Rubinstein norm and the space $(\operatorname{lip}(K, \rho))^{*}$ or equivalently between the spaces $\operatorname{Lip}(K, \rho)$ and $(\operatorname{lip}(K, \rho))^{* *}$. Such metric spaces are studied and related properties of Lipschitz spaces are established.
\end{abstract}

\section{Notation}

Let $(K, \rho)$ be a metric space and $M(K)$ be the set of all finite Borel measures on $K$. For a measure $\mu \in M(K)$, denote by $\mu_{+}, \mu_{-}$its positive and negative variations, respectively, and set $|\mu|=\mu_{+}+\mu_{-}, \operatorname{Var} \mu=|\mu|(K)$.

The Lipschitz space $\operatorname{Lip}(K, \rho)$ is defined as the set of all functions $f$ on $K$ with the finite norm

$$
\|f\|_{K, \rho}=\max \left\{\|f\|_{K},|f|_{K, \rho}\right\},
$$

where

$$
\|f\|_{K}=\sup \{|f(x)|: x \in K\}
$$

and

$$
|f|_{K, \rho}=\sup \left\{\frac{|f(x)-f(y)|}{\rho(x, y)}: x, y \in K, x \neq y\right\} .
$$

Functions $f \in \operatorname{Lip}(K, \rho)$ with the property

$$
\lim _{\rho(x, y) \rightarrow 0} \frac{f(x)-f(y)}{\rho(x, y)}=0
$$

constitute the closed subspace $\operatorname{lip}(K, \rho)$ in $\operatorname{Lip}(K, \rho)$. The notation $B$ and

Received by the editors July 13, 1990.

1991 Mathematics Subject Classification. Primary 46E15, 46E27; Secondary 28A33, 54E35.

Key words and phrases. Kantorovich-Rubinstein norm, Lipschitz space, completion, isometric isomorphism. 
$b$ will be used for closed unit balls in $\operatorname{Lip}(K, \rho)$ and $\operatorname{lip}(K, \rho)$, respectively. All spaces of measures and functions below are supposed to be real.

\section{INTRODUCTION AND BACKGROUND}

Let $(K, \rho)$ be a compact metric space. The total variation norm Var on $M(K)$ suffers from at least two grave shortcomings:

(i) it is very weakly connected with the metric since it is invariant for all metrics generating the same topology;

(ii) there is no transparent description of the conjugate space $(M(K))^{*}$.

This suggests the question: can another norm on $M(K)$ that is free from these defects be defined? Such norm has been actually invented in 1942 by Kantorovich [4] and has been studied in his works with Rubinstein [6, 7]. This norm is called the Kantorovich-Rubinstein (KR) norm.

For distinct points $x, y \in K$, the KR norm of the dipole $\delta_{x}-\delta_{y}$ is $\rho(x, y)$, while $\operatorname{Var}\left(\delta_{x}-\delta_{y}\right)=2$. Thus, for each infinite set $K$, the space $M(K)$ with the KR norm is not complete.

The present work contains a theorem providing a description of its completion and connected results on Lipschitz spaces.

Let us recall the definition of the original KR norm (for this and other information on KR theory see [5, Chapter VIII, §4]). Let $M_{0}(K)$ be the set of measures $\mu \in M(K)$ such that $\mu(K)=0$. With each measure $\mu \in M_{0}(K)$, we associate the family $\Psi_{\mu}$ of all nonnegative measures $\Psi \in M(K \times K)$ such that for every Borel set $e \subset K, \Psi(K, e)-\Psi(e, K)=\mu(e)$. The value $\Psi\left(e_{1}, e_{2}\right)$ can be interpreted as the mass carried from a set $e_{1}$ to a set $e_{2}$. Thus $\psi \in \Psi_{\mu}$ gives rise to a mass transfer on $K$ with the given mass distribution $\mu_{-}$and the required one $\mu_{+}$. The classical $\mathrm{KR}$ norm of a measure $\mu \in M_{0}(K)$ is defined by

$$
\|\mu\|_{\rho}^{0}=\inf \left\{\int_{K \times K} \rho(x, y) d \psi(x, y): \psi \in \Psi_{\mu}\right\} .
$$

Its value together with the corresponding optimal transfer give the solution of the Monge-Kantorovich mass transfer problem [5, 10].

Now set

$$
\|\mu\|_{\rho}=\inf \left\{\|\nu\|_{\rho}^{0}+\operatorname{Var}(\mu-\nu): \nu \in M_{0}(K)\right\} \quad(\mu \in M(K)) .
$$

The functional $\|\cdot\|_{\rho}$ is a norm on $M(K)$. Though it differs from the norm on $M(K)$ introduced in [6], we preserve the name KR norm for it. Since

$$
\|\mu\|_{\rho}^{0} \leq \frac{1}{2} \operatorname{diam}(K, \rho) \operatorname{Var} \mu \quad\left(\mu \in M_{0}(K)\right),
$$

the norms $\|\cdot\|_{\rho}$ and $\|\cdot\|_{\rho}^{0}$ on $M_{0}(K)$ are equivalent (if $\operatorname{diam}(K, \rho) \leq 2$, they even coincide).

The following theorem (analogous to its counterparts in [5] and [7]) is the main result of the KR theory.

Theorem 0. The duality

$$
\langle f, \mu\rangle=\int_{K} f d \mu \quad(\mu \in M(K), f \in \operatorname{Lip}(K, \rho))
$$


establishes an isometric isomorphism between the spaces $\left(M(K),\|\cdot\|_{\rho}\right)^{*}$ and $\operatorname{Lip}(K, \rho)$.

This result depends largely on the following basic property of the KR norm (compare with the corresponding statement in [5]).

Lemma 0. The set of all measures with finite support is dense in $M(K)$ with respect to the KR norm.

\section{Statement of the Results}

Let $(K, \rho)$ be a compact metric space. For every $\mu \in M(K)$, the formula

$$
i(\mu)(f)=\int_{K} f d \mu \quad(f \in \operatorname{lip}(K, \rho))
$$

defines a bounded linear functional $i(\mu)$ on $\operatorname{lip}(K, \rho)$.

Lemma 1. The set $i(M(K))$ is dense in $(\operatorname{lip}(K, \rho))^{*}$ in the norm topology.

For $K=[0,1], \rho(x, y)=|x-y|^{\alpha}, 0<\alpha<1$, the proof is contained in [8, Lemma 2.5]. In the more general case when $(K, d)$ is a compact metric space and $\rho=d^{\alpha}, 0<\alpha<1$, it is established in a similar way in [1, Lemma 3.1]. In the case of arbitrary compact metric space $(K, \rho)$, the proof is the same.

By means of Theorem 0, we have

$$
|i(\mu)(f)| \leq\|f\|_{K, \rho}\|\mu\|_{\rho} \quad(f \in \operatorname{lip}(K, \rho), \mu \in M(K)) .
$$

Hence $i:\left(M(K),\|\cdot\|_{\rho}\right) \rightarrow(\operatorname{lip}(K, \rho))^{*}$ is a linear map with the norm $\leq$ 1. Passing to the completion, we extend $i$ to a map $i:\left(M(K),\|\cdot\|_{\rho}\right)^{c} \rightarrow$ $(\operatorname{lip}(K, \rho))^{*}$ with the same properties (and notation).

The question we start with is the following: Under what conditions is the above map an isometric isomorphism?

Theorem 1. The map $i:\left(M(K),\|\cdot\|_{\rho}\right)^{c} \rightarrow(\operatorname{lip}(K, \rho))^{*}$ is an isometric isomorphism if and only if the following condition is satisfied:

for every finite set $F \subset K$ and every function $f$ on $F$, for each

$C>1$ there exists a function $g \in \operatorname{lip}(K, \rho)$ such that $g \mid F=f$ and $\|g\|_{K, \rho} \leq C\|f\|_{F, \rho}$.

Remark. A closed interval with the Euclidean metric (or every metric space containing it as a subspace) fails to have property (A).

Taking the conjugate map $i^{*}$ and applying Theorem 0 we get the natural linear map $j:(\operatorname{lip}(K, \rho))^{* *} \rightarrow \operatorname{Lip}(K, \rho)$ with the norm $\leq 1$. Then, from Theorem 1, we obtain the following result.

Theorem 2. The map $j:(\operatorname{lip}(K, \rho))^{* *} \rightarrow \operatorname{Lip}(K, \rho)$ is an isometric isomorphism if and only if the metric space $(K, \rho)$ satisfies condition (A).

The following definition is intended to provide a condition stronger than (A) but expressed in terms of metrics.

Definition 1. A metric $\rho$ on a set $K$ is called noncritical if there is a sequence $\left\{\rho_{n}\right\}_{n \in \mathbb{N}}$ of metrics on $K$ such that

(i) $\lim _{n \rightarrow \infty} \rho_{n}(x, y)=\rho(x, y)$ for all $x, y \in K$;

(ii) $\lim _{n \rightarrow \infty} \sup \left\{\rho_{n}(x, y) / \rho(x, y): x, y \in K, x \neq y\right\}=1$;

(iii) $\lim _{\rho(x, y) \rightarrow 0} \rho_{n}(x, y) / \rho(x, y)=0$ for all $n \in \mathbb{N}$. 
Proposition 1. Every noncritical (not necessarily compact) metric possesses prop$\operatorname{erty}(\mathrm{A})$.

Now we will introduce a more constructively defined subclass of noncritical metrics.

Definition 2. Let $\Omega$ be the set of all nondecreasing functions $\omega: \mathbb{R}_{+} \rightarrow \mathbb{R}_{+}$ such that $\omega(0)=0, \lim _{t \rightarrow 0} \omega(t)=0, \lim _{t \rightarrow 0} \omega(t) / t=+\infty$, and the function $\omega(t) / t$ is nonincreasing for $t>0$.

The latter condition implies that every function $\omega \in \Omega$ is semiadditive, i.e., $\omega(s+t) \leq \omega(s)+\omega(t)$ for all $s, t \geq 0$. Hence, if $\rho$ is a (compact) metric then the same is $\omega(\rho)$ for all $\omega \in \Omega$.

Proposition 2. If $d$ is a metric on a set $K$ then, for every $\omega \in \Omega, \rho=\omega(d)$ is a noncritical metric on $K$.

Thus, for every compact noncritical metric (in particular, for a metric of the form $\rho=\omega(d), \omega \in \Omega$, we have isometric isomorphisms indicated in Theorems 1 and 2. In the particular case $\omega(t)=t^{\alpha}, 0<\alpha<1$, an isometric isomorphism between the spaces $(\operatorname{lip}(K, \omega(d)))^{* *}$ and $\operatorname{Lip}(K, \omega(d))$ has been established earlier. For $K=[0,1], d(x, y)=|x-y|$, this result has been originally obtained in [8], and for arbitrary compact metric space $(K, d)$ in [2, $3]$. In the latter case, the above isomorphism has been also rediscovered in [1].

For metrics of the form $\rho=\omega(d), \omega \in \Omega$, property (A) can be strengthened.

Proposition 3. Let $(K, d)$ be a metric space, and let $\rho=\omega(d)$ with $\omega \in \Omega$. Then for every finite set $F \subset K$ and every function $f$ on $F$, for each $C>$ 1 there exists a function $g \in \operatorname{Lip}(K, d)$ such that $g \mid F=f$ and $\|g\|_{K, \rho} \leq$ $C\|f\|_{F, \rho}$.

The following Stone-Weierstrass type theorem for the spaces $\operatorname{lip}(K, \rho)$ is connected with Proposition 3.

Theorem 3. Let $(K, \rho)$ be a compact metric space. Suppose $L$ is a linear subspace of $\operatorname{lip}(K, \rho)$ satisfying the following condition: there is a constant $C$ such that for every finite set $F \subset K$ and for every function $f \in \operatorname{lip}(K, \rho)$ there exists a function $g \in L$ with the properties $g|F=f| F$ and $\|g\|_{K, \rho} \leq C\|f\|_{K, \rho}$. Then $L$ is dense in $\operatorname{lip}(K, \rho)$.

From Theorem 3 and Proposition 3 we obtain

Proposition 4. Suppose $(K, d)$ is a compact metric space, and let $\rho=\omega(d)$, $\omega \in \Omega$. Then the space $\operatorname{Lip}(K, d)$ is dense in $\operatorname{lip}(K, \rho)$.

For compact metric spaces $(K, \rho)$ with $\rho=d^{\alpha}, 0<\alpha<1$, Proposition 3 (with $C=\sqrt{2}$ ), Theorem 3, and Proposition 4 can be found in [1].

\section{Proofs}

Proof of Theorem 1. Necessity. We suppose that $i$ is an isometric isomorphism, and we shall show that condition (A) is fulfilled. Let $F$ be an $n$-point subset in $K$. Set $E=\{g \mid F: g \in \operatorname{lip}(K, \rho)\}$. Obviously, $E$ can be identified with a linear subspace in $\mathbb{R}^{n}$. We claim that $E=\mathbb{R}^{n}$. To see this, take a functional 
annihilating $E$. This functional can be viewed as a measure $\mu$ on $F$ (and thus on $K$ ). Then

$$
\int_{K} g d \mu=0 \quad(g \in \operatorname{lip}(K, \rho)) .
$$

It means that $i(\mu)=0$. Since the map $i$ is supposed to be one-to-one, this implies that $\mu=0$, and hence that $E=\mathbb{R}^{n}$.

Set $X=\{g \in \operatorname{lip}(K, \rho): g \mid F=0\}$ and observe that the set of functionals in $(\operatorname{lip}(K, \rho))^{*}$ annihilating $X$ coincides with $i(M(F))$. Let $f$ be a function on $F$. As was shown earlier, there is a function $g_{0} \in \operatorname{lip}(K, \rho)$ such that $g_{0} \mid F=f$. Since $i$ is an isometry, by Theorem 0 we have

$$
\begin{aligned}
\operatorname{dist}\left(g_{0}, x\right) & =\sup \left\{\lambda\left(g_{0}\right): \lambda \in(\operatorname{lip}(K, \rho))^{*}, \lambda(X)=\{0\},\|\lambda\|=1\right\} \\
& =\sup \left\{\int_{F} f d \mu: \mu \in M(F),\|\mu\|_{\rho}=1\right\}=\|f\|_{F, \rho},
\end{aligned}
$$

where by $\|\cdot\|$ is denoted hereafter the usual norm on $(\operatorname{lip}(K, \rho))^{*}$. Hence for every $C>1$ we can find a function $g_{1} \in X$ such that $\left\|g_{0}-g_{1}\right\|_{K, \rho} \leq C\|f\|_{F, \rho}$. Then $g=g_{0}-g_{1}$ is the function required.

Sufficiency. Suppose that condition (A) is satisfied. We need to show that the map $i$ is an isometry. To see this, take $\mu \in M(K), f \in B$, and $\varepsilon>0$. By Lemma 0 there exists a measure $\nu$ on $K$ with finite support $F$ such that $\|\nu-\mu\|_{\rho} \leq \varepsilon$. Condition (A) supplies us with a function $g \in \operatorname{lip}(K, \rho)$ with $g|F=f| F$ and $\|g\|_{K, \rho} \leq 1+\varepsilon$. Then via Theorem 0

$$
\int_{K} f d \mu=\int_{K} f d(\mu-\nu)+\int_{K} g d(\nu-\mu)+\int_{K} g d \mu \leq(2+\varepsilon) \varepsilon+\int_{K} g d \mu .
$$

Hence again by means of Theorem 0 we obtain

$\|\mu\|_{\rho}=\sup \left\{\int_{K} f d \mu: f \in B\right\}=\sup \left\{\int_{K} g d \mu: g \in b\right\}=\|i(\mu)\| \quad(\mu \in M(K))$.

Proof of Proposition 1. Let $\rho$ be a noncritical metric on $K$, and let $\left\{\rho_{n}\right\}_{n \in \mathbb{N}}$ be a sequence of metrics satisfying conditions (i)-(iii) of Definition 1. Take a finite set $F$ in $K$ and a function $f$ on $F$. Denote

$$
\begin{aligned}
& \alpha_{n}=\max \left\{\frac{\rho(x, y)}{\rho_{n}(x, y)}: x, y \in F, x \neq y\right\}, \\
& \beta_{n}=\sup \left\{\frac{\rho_{n}(x, y)}{\rho(x, y)}: x, y \in K, x \neq y\right\} \quad(n \in \mathbb{N}) .
\end{aligned}
$$

It follows from conditions (i) and (ii) that $\alpha_{n} \rightarrow 1$ and $\beta_{n} \rightarrow 1$ as $n \rightarrow \infty$. Note that $\|f\|_{F, \rho_{n}} \leq \max \left\{\alpha_{n}, 1\right\}\|f\|_{F, \rho}$. The function $f$ can be extended to a function $f_{n} \in \operatorname{Lip}\left(K, \rho_{n}\right)$ in such a way that $\left\|f_{n}\right\|_{K, \rho_{n}}=\|f\|_{F, \rho_{n}}$ [9]. Condition (iii) implies $f_{n} \in \operatorname{lip}(K, \rho)$. Further,

$$
\left\|f_{n}\right\|_{K, \rho} \leq \max \left\{\beta_{n}, 1\right\}\left\|f_{n}\right\|_{K, \rho_{n}} \leq \max \left\{\beta_{n}, 1\right\} \max \left\{\alpha_{n}, 1\right\}\|f\|_{F, \rho} \quad(n \in \mathbb{N}) .
$$

Then, for every $C>1$, the function $g=f_{n}, n$ being sufficiently large, meets condition (A). 
Proof of Proposition 2. Suppose $d$ is a metric on a set $K, \omega \in \Omega$, and $\rho=$ $\omega(d)$. For every $n \in \mathbb{N}$, define the function

$$
\varphi_{n}(t)= \begin{cases}0 & \text { if } t=0 ; \\ \left(n \omega\left(\frac{1}{n}\right) \frac{t}{\omega(t)}\right)^{1 / 2} & \text { if } 0<t<\frac{1}{n} \\ 1 & \text { if } t \geq \frac{1}{n} .\end{cases}
$$

It can be seen easily that the functions $\omega_{n}=\varphi_{n} \omega$ belong to $\Omega$ for all $n$. The sequence of functions $\left\{\varphi_{n}\right\}_{n \in \mathbb{N}}$ possesses the following properties:

(i) $\lim _{n \rightarrow \infty} \varphi_{n}(t)=1$ for all $t>0$;

(ii) $\sup _{t>0} \varphi_{n}(t)=1$ for all $n \in \mathbb{N}$;

(iii) $\lim _{t \rightarrow 0} \varphi_{n}(t)=0$ for all $n \in \mathbb{N}$.

Then the corresponding sequence of metrics $\rho_{n}=\omega_{n}(\rho)=\varphi_{n}(\rho) \rho,(n \in \mathbb{N})$ satisfies Definition 1. Thus $\rho$ is noncritical metric.

Proof of Proposition 3. Let $d$ be a metric on a set $K$, and let $\rho=\omega(d)$ where $\omega \in \Omega$. Take a finite set $F \subset K$, a function $f$ on $F$, and arbitrary $C>1$. Denote $\delta_{0}=\min \{\rho(x, y): x, y \in F, x \neq y\}$, and choose $\delta \in\left(0, \delta_{0}\right)$ such that $\delta=\omega(\tau)$ for some $\tau>0$ and

$$
\max _{x, y \in F} \frac{\rho(x, y)}{\rho(x, y)-\delta} \leq C .
$$

For $x, y \in K$, define $r(x, y)=\max \{\rho(x, y)-\delta, 0\}$.

Note that if there is a nonvoid family of functions $\left\{f_{\alpha}: \alpha \in \mathscr{A}\right\}$ defined on a metric space $(X, \sigma)$ and satisfying for some $N$ and for all $x, y \in X$ the Lipschitz condition

$$
f_{\alpha}(x)-f_{\alpha}(y) \leq N \sigma(x, y) \quad(\alpha \in \mathscr{A}),
$$

then the same is true for the function $\sup \left\{f_{\alpha}(x): \alpha \in \mathscr{A}\right\},(x \in X)$ provided that the latter is not identically $+\infty$.

Applying this, we see that for every $y \in K$,

$$
r(x, y)-r(z, y) \leq \rho(x, z) \quad(x, z \in K) .
$$

Set $M=\|f\|_{F, \rho}$ and define the function

$$
h(x)=\sup \{f(y)-C M r(x, y): y \in F\} \quad(x \in K) .
$$

We claim that $h \mid F=f$. Clearly, $h(x) \geq f(x),(x \in F)$. Conversely, for given $x \in F$ we have, for all $y \in F \backslash\{x\}$,

$$
f(y)-f(x) \leq M \rho(x, y) \leq C M(\rho(x, y)-\delta)=C M r(x, y),
$$

and hence $f(x) \geq h(x)$.

It follows from (1) that, for every $y \in F$, the function $x \mapsto f(y)-C M r(x, y)$ satisfies the Lipschitz condition with respect to the metric $\rho$ with the constant $C M$. Using the note mentioned above, we obtain $|h|_{K, \rho} \leq C\|f\|_{F, \rho}$.

Now we will show that there is a constant $P$ such that for every $y \in K$,

$$
r(x, y)-r(z, y) \leq P d(x, z) \quad(x, z \in K) .
$$


It is sufficient to demonstrate this inequality for $\rho(x, y) \geq \delta$ and $r(z, y) \leq$ $r(x, y)$. If $\rho(z, y)<\delta$ then $d(z, y) \leq \tau$, while $d(x, y) \geq \tau$. In this case, we have

$$
\begin{aligned}
r(x, y)-r(z, y) & =\rho(x, y)-\delta=\omega(d(x, y))-\omega(\tau) \\
& \leq \frac{\omega(\tau)}{\tau}(d(x, y)-\tau) \leq \frac{\omega(\tau)}{\tau}(d(x, y)-d(z, y)) \\
& \leq \frac{\omega(\tau)}{\tau} d(x, z)
\end{aligned}
$$

If $\rho(z, y) \geq \delta$ then $d(z, y) \geq \tau$, and in this case, we have

$$
\begin{aligned}
r(x, y)-r(z, y) & =\rho(x, y)-\rho(z, y) \\
& \leq \frac{\omega(d(z, y))}{d(z, y)}(d(x, y)-d(z, y)) \leq \frac{\omega(\tau)}{\tau} d(x, z) .
\end{aligned}
$$

Thus (2) is valid with $P=\omega(\tau) / \tau$. Again applying the "supremum argument" to the function $h$ we obtain via (2) that $h \in \operatorname{Lip}(K, d)$. Finally,

$$
g(x)= \begin{cases}M & \text { if } h(x)>M \\ h(x) & \text { if }|h(x)| \leq M \\ -M & \text { if } h(x)<-M\end{cases}
$$

is the function desired.

Proof of Theorem 3. We have to show that for every functional $\varphi \in(\operatorname{lip}(K, \rho))^{*}$ annihilating $L, \varphi=0$. Take $f \in \operatorname{lip}(K, \rho)$ and $\varepsilon>0$. By Lemma 1 there is a measure $\mu \in M(K)$ such that $\|i(\mu\|i(\mu)-\varphi\| \leq \varepsilon)-\varphi\| \leq \varepsilon$, and by Lemma 0 we can find a measure $\nu$ on $K$ with a finite support $F$ such that $\|\nu-\mu\|_{\rho} \leq \varepsilon$. Hence $\|i(\nu)-\varphi\| \leq 2 \varepsilon$ since the norm of $i$ is not greater than 1. There exists a function $g \in L$ with $g|F=f| F$ and $\|g\|_{K, \rho} \leq C\|f\|_{K, \rho}$. We have $\varphi(f)=(\varphi-i(\nu))(f)+(i(\nu)-\varphi)(g)$; then $|\varphi(f)| \leq 2 \varepsilon(1+C)\|f\|_{K, \rho}$. This implies that $\varphi(f)=0(f \in \operatorname{lip}(K, \rho))$, i.e., $\varphi=0$.

\section{ACKNOWLEDGMENTS}

It gives me great pleasure to express my gratitude to Professor G. Sh. Rubinstein for useful discussion. I am thankful to Professor Yu. A. Brudnyi for his helpful advice. I am grateful to Professor I. E. Ovcharenko for his encouraging enthusiasm about the KR norm. I would like also to thank the referee for stylistic improvement of the manuscript.

\section{REFERENCES}

1. W. G. Bade, P. C. Curtis, Jr., and H. G. Dales, Amenability and weak amenability for Beurling and Lipschitz algebras, Proc. London Math. Soc. (3) 55 (1987), 359-377.

2. T. M. Jenkins, Banach spaces of Lipschitz functions on an abstract metric space, Thesis, Yale Univ., New Haven, CT, 1967.

3. J. A. Johnson, Banach spaces of Lipschitz functions and vector-valued Lipschitz functions Bull. Amer. Math. Soc. 75 (1969), 1334-1338.

4. L. V. Kantorovich, On mass transfer, Dokl. Akad. Nauk SSSR 37 (1942), 227-229. (Russian)

5. L. V. Kantorovich and G. P. Akilov, Functional analysis, 2nd ed., New York, 1982. 
6. L. V. Kantorovich and G. Sh. Rubinstein, On a functional space and certain extremal problems, Dokl. Akad. Nauk SSSR 115 (1957), 1058-1061. (Russian)

7. __ On a space of completely additive functions, Vestnik Leningrad Univ. Math. 13 (1958), 52-59. (Russian)

8. K. de Leeuw, Banach spaces of Lipschitz functions, Studia Math. 21 (1961), 55-66.

9. E. J. McShane, Extension of range of functions, Bull. Amer. Math. Soc. 40 (1934), 837-842.

10. S. T. Rachev, The Monge-Kantorovich mass transference problem and its stochastic applications, Teor. Veroyatnost. i Primenen. 29 (1984), 625-653 (Russian); English transl. in Theor. Probab. Appl. 29 (1984), 647-676.

InstitutskiJ Prospect 29, APt. 45, St. Petersburg, 194021, Russia

Current address: Department of Mathematics, The Technion-Israel Institute of Technology, Haifa 32000, Israel

E-mail address: MAR9317@TECHNION.BITNET 